\title{
El Conocimiento Animal
}

\section{Gnostic Processes in Animals}

\author{
Guy Santibáñez-H. ${ }^{1}$ \\ Juan E. Dominichetti C. ${ }^{2}$ \\ Marcelo Sanhueza Z. ${ }^{3}$
}

\begin{abstract}
Resumen
Al comparar las funciones psicofisiológicas que subtienden la adaptación en animales no humanos con las correspondientes del Homo sapiens se ha llegado a la conclusión que los procesos son análogos. Los animales no solo son capaces de comportarse, sino de presentar fenómenos subjetivos y operar con conocimientos.

Hay tres funciones llamadas "funciones integrativas del cerebro" que subtienden las funciones mencionadas.

Los primates tienen increíbles capacidades; pueden operar con el lenguaje de sordos-mudos, y son capaces de aprender un lenguaje diseñado especialmente para ellos sobre bases computacionales, que se ha llamado "Yerkes". Este lenguaje permite comunicación entre el investigador y el animal, o entre dos animales.

Los primates no humanos tienen varias normas que rigen su vida social, desarrollan actividades lúdicas, usan herramientas y descubren soluciones a problemas complejos.

Diversas especies animales aprenden por imitación, obedecen órdenes, reaccionan a diversas señales de sus congéneres.

En este trabajo nos referimos a estos fenómenos subjetivos, y a los conocimientos que integran el conjunto de procesos psicofisiológicos de los animales no humanos.

Se discute el significado adaptativo de estas funciones y algunos trabajos recientes que muestran que ciertas habilidades gnósticas tienen un efecto productor de neogénesis neuronal en vertebrados adultos.
\end{abstract}

Palabras claves: Cerebro, subjetividad, conocimiento, neogénesis neuronal.

\begin{abstract}
A comparison of the psycho physiological functions that underlie adaptation in nonhuman animals with the corresponding ones in Homo sapiens leads to the conclusion that they are analogous. Non human animals are not only capable of behaving, but they also exhibit subjective phenomena and are able to operate with knowledge.
\end{abstract}

1 Profesor Titular, Departamento de Psicología, Universidad de Chile.

2 Ayudante, Departamento de Psicología, Universidad de Chile.

3 Ayudante, Departamento de Psicología, Universidad de Chile. 
The above mentioned functions are based on three so called "integrative activities of the brain".

Primates possess incredible abilities: individuals from some species can operate with a language designed for deaf-mutes, and are also able to learn a computationally based language designed especially for them, called "Yerkes". This language allows communication between the researcher and the animals and also between two animals.

Many non human primate societies are governed by strict norms, they perform play activities, they use tools and discover solutions for complex problems.

Members of many animal species learn my imitation, obey orders, react to signals from their co specifics.

In this paper we refer to these subjective phenomena and to the factors that integrate the set of psycho physiological processes in these non human animals.

We discuss the adaptive significance of these functions and review some recent studies that reveal that certain Gnostic abilities lead to neogenesis of neurons in adult vertebrates.

Keywords: brain, subjectivity, knowledge, neural neogenesis.

\section{Introducción}

Los animales, sin discusión tienen, aún en los niveles más humildes de la evolución, comportamiento. En la cultura grecolatina ha predominado la idea de atribuir la propiedad de conocer, de razonar, de discernir, de una manera predominante, al Homo sapiens. Esto como una consecuencia necesaria de poseer una "alma" adecuada para llevar a cabo las operaciones intelectuales mencionadas.

La discusión ha cambiado de centro gravitacional. Los procesos subjetivos han sido calificados genéricamente como "cognición". Esta palabra es etimológicamente inadecuada puesto que los procesos subjetivos pueden tener relaciones con procesos gnósticos provenientes del medio externo; con actividad proveniente del medio interno o simplemente con elementos que se generan directa o indirectamente en el medio subjetivo. Algunos de estos fenómenos constituyen conocimientos, otros no, como por ejemplo delirios, alucinaciones, creencias mágicas, etc.
Recordemos algunos autores A.E. Brehms el celebre autor de su famosa "Vida de los Animales". ("Tierleben" publicada en 1863) donde analiza las condiciones de vida de diversas especies y hace observaciones sobre sus comportamientos. J.H. Fabre, quien en sus "Recuerdos entomológicos" (1946) hace magnificas descripciones de los comportamientos de los insectos.

A comienzo del siglo XX hay una gran cantidad de trabajos dedicados a la Psicología Animal. H. Piéron (1941) hace una síntesis de los conocimientos en ésta área destacando el significado de los procesos instintivos y aprendidos, Guillaume (1947) se refiere al instinto y a la inteligencia, Buytendjik (1928) discute el pensamiento animal donde analiza un famoso trabajo de K. Krall ("Denkende tiere" - El animal pensante, 1912).

El magnífico trabajo de Warden, Jenkins y Warner (1940) ha sido una gran contribución no sólo al conocimiento de la psicología animal sino también a poner de relieve las diferencias entre las especies, tanto en lo que se refiere a las funciones que se comparan, como a la evolución de ella. 
En el trabajo de los autores citados se estudian las diferentes aspectos psicológicos, integrando conocimiento de fisiología psicológica, psicología experimental, de psicología del aprendizaje y otros aspectos como la adaptación.

La experimentación con diferentes técnicas ha permitido conocer como los animales aprenden, es decir como adquieren conocimientos en condiciones metodológicamente controladas, ha contribuido decididamente a aumentar el caudal de conocimientos acerca de los procesos gnósticos en las diversas especies.

Hay todavía un tercer elemento que ha influido en el progreso del conocimiento de los fenómenos gnósticos: la remoción de los prejuicios sobre las diferencias excluyentes entre animales y el Homo sapiens con respecto a sus respectivas habilidades de aprendizaje. El centro del interés en el estudio de la génesis del conocimiento se ha desplazado desde el "alma" a las funciones cerebrales. I.M. Sechenov 1863 (1965) describe de una manera magistral un movimiento voluntario señalando sus diferencias con un reflejo involuntario, automático. Sostiene que esta transformación implica una interesante interacción entre fenómenos excitatorios e inhibitorios, además de la activación subjetiva. Bautizó este tipo de reflejo como "reflejos del cerebro". El encuentro de Pavlov con un curioso fenómeno, la activación de la secreción salival de un perro adulto por el contacto visual con un pedazo de carne y la comprobación que esta reacción no se producía en los animales sin previa experiencia alimenticia con carne, despertó en él un gran interés. Llamó a éste fenómeno "secreción salival psíquica". Esto fue comunicado por primera vez 1895 (Pawlow, 1953). Algunos años mas tarde en una conferencia en Londres hizo una contribución con el titulo "Investigaciones científicas naturales de los así llamados actividades del alma de los animales superiores" (Pawlow, 1953). Fischel (1961) dedica un trabajo a la discusión del tema.
Pavlov siguiendo el camino de Setchenov señala la necesidad de integrar los procesos psíquicos con las funciones del cerebro. Konorski (1948) a su vez escribió en la dedicatoria su libro a "I.P.Pavlov y C.S. Sherrington en la esperanza que este trabajo hará algo para llenar el vacío que existe entre sus logros".

Konorski (1967) entrega una síntesis de los logros que el alcanzó en el camino recorrido.

La integración de todos estos enfoques está permitiendo la obtención de conocimientos acerca de los procesos gnósticos.

\section{Como se estudia el conocimiento animal}

El problema del conocimiento se puede abordar en una serie de diversas interacciones entre el animal, tomado como sujeto de estudio, y el medio con el cual este sujeto interactúa.

Hay dos posibles condiciones generales. El animal se encuentra en su medio natural o bien en un medio que puede serle ajeno en diversos grados. Esto significa que puede ser estudiado en un medio que no es el suyo. En cautiverio como por ejemplo en un jardín zoológico o en condiciones de domesticación, es decir en armonía con el medio doméstico y sus habitantes. Las condiciones de laboratorio están entre la cautividad y la domesticación.

Ahora bien la técnica de trabajo empleada en condiciones naturales es, fundamentalmente la observación. Sea directa, en la cual el investigador percibe directamente algunos aspectos del comportamiento del animal observado. El comportamiento, descrito como la figura efectiva perceptible por un observador es simplemente una parte del modelo efector que incluye también el medio interno. No hay reacciones musculares sin un compromiso de algún componente viscero-endo- 
crino controlado por la porción correspondiente del encéfalo. En la actualidad es posible poder observar el registro de la actividad de algunas vísceras aunque el sujeto no esté al alcance de los receptores del investigador. A distancia se puede controlar aún la actividad electromagnética del encéfalo.

La vida en común entre animales y seres humanos puede ser de una gran utilidad para ambos. La interacción permanente, la adaptación recíproca, determina un incremento del conocimiento recíproco. En estas condiciones se hacen evidentes fenómenos en los cuales están envueltos procesos subjetivos y comportamientos, actividades intelectuales y emocionales, modulaciones excitatorios e inhibitorios, etc.

Los experimentos han puesto a la Psicología en el camino de operar con conocimientos más sólidos que los suministrados por la metafísica y las posturas ideológicas. Las técnicas que usa la Psicología en su desarrollo experimental, incluyen aquellas que permiten usar instrumentos para entrenar animales y personas, establecer correlaciones entre la actividad del medio interno y el comportamiento, estudiar las funciones cerebrales envueltas sea con técnicas de extirpación o de registro eléctrico, sea con las técnicas de emisión de positrones y de registro funcionales de las imágenes de resonancia magnética.

\section{Algunos logros de la psicología en el conocimiento de los procesos gnósticos}

Al analizar la evolución de las Ciencias Psicológicas se observa un cambio en la acentuación de los procesos que han sido considerados como los temas predominantes en la preocupación de estas ciencias El nombre de esta ciencia proviene de la palabra griega que significa "alma". En este contexto las reacciones comportamentales eran producto de las "decisiones" del alma. Por lo tanto lo significativo era el "alma", instancia que de alguna manera habría tenido algo que ver con el cerebro, al menos como su alojamiento.

Mientras los filósofos helénicos admitían que animales y aún plantas tenían algún tipo de alma, el principio activador del cuerpo estaría presente en todo ser viviente (El anima de Aristóteles).

El razonable Descartés era de la opinión que alma es una posesión puramente humana. Los animales, en cambio, eran autómatas sin alma. Esta mareante diferencia abría solamente al Homo sapiens la posibilidad de "pensar" o si se quiere "reflexionar".

Las contribuciones de Wundt (1874) introdujeron la interacción "comportamientocerebro" a la palestra científica, y los trabajos de Sechenov (1863) y Pavlov contribuyeron a desarrollar la concepción de reflejos psíquicos o del encéfalo o condicionados. Restableciendo así la interacción funcional entre los procesos subjetivos y las reacciones corporales. Sin embargo durante el siglo XX la Psicología se ha ocupado del comportamiento que ha sido tratado experimentalmente, mientras los procesos subjetivos han obtenido una consideración fundamentalmente reflexiva o especulativa.

A partir de la aparición de una psicología que ha tomado como objetivo exclusivo el "comportamiento", han sido excluidos del estudio dos fenómenos básicos: el rol que juega el sistema Neuroendocrino en la integración de diversos elementos del comportamiento y por otra parte el significado que tienen los procesos subjetivos en la adaptación de los individuos de diversas especies a sus respectivos loci ambientales.

Por otra parte resulta evidente que el locus orgánico responsable tanto del comportamiento como de los procesos subjetivos es el sistema Neuroendocrino, cuyo centro de integración es el Encéfalo. En la actualidad hay evidencias experimentales y clínicas, tanto en el Homo sapiens como en otras especies, que comportamiento y pro- 
cesos subjetivos son funciones de grupos de neuronas que trabajan organizada e integradamente en el cerebro. ( Gazzaniga 1995). Se ha distinguido desde una perspectiva psicofisiológica lo que Konorski ha llamado funciones integrativas del cerebro o del sistema neuroendocrino (Konorski 1967). Entendiéndose por "integración neural" el trabajo conjunto de diversos sistemas neuronales los cuales constituyen la base funcional de la adaptación de los individuos de diversas especies a sus respectivos medios.

Estos procesos integrativos generarían tres conjuntos funcionales: las funciones reactivas genéticamente determinadas, las funciones plásticas aprendidas a través del contacto de los individuos con los medios objetivos (medio externo y medio interno) y las funciones subjetivas las cuales conforman los procesos virtuales que emergen de la asimilación de la experiencia individual (Konorski 1967, Santibáñez - H, 1981, 1986).

Las funciones subjetivas pueden jugar un rol en los procesos plásticos de los animales, como propuso Thorndike, en el aprendizaje, por ensayo y error. (Thorndike, 1911) y en los procesos de "directa comprensión" (insight-"einsicht") como describió Köhler (1925) en su conocido trabajo con primates.

Krech (1932) describió que ratas entrenadas en pruebas de ensayo y error consideraban ciertas claves como más significativas que otras. Mientras en pruebas discriminación, las estrategias de aprendizaje oscilaban alrededor del nivel del azar, si se tomaban en consideración otras claves, diferentes de la discriminada, el nivel era diferente del azar. Las ratas se comportaban "como si" fueran probando claves hipotéticas diferentes a las esperadas por el investigador. El comportamiento de "ensayo y error" con respecto a un sistema de claves puede ser sistemático con respecto a otras claves. Krech y Crutchfieldd (1948) postularon que el proceso de aprendizaje puede ser considerado como una "reorganización del campo gnóstico".
Se ha intentado enseñar a primates a utilizar formas de comunicación utilizando las técnicas humanas. K. y C. Hayes (1971), intentaron enseñar a pronunciar palabras a un chimpancé. Después de denodados esfuerzos lograron que el animalito dijera algunas palabras como "mamá", " papá", "taza". Los chimpancés son capaces imitar los gestos humanos, habilidad que fue utilizada por Allen y Beatriz Gardner (1969) para entrenar a un chimpancé (Washoe), luego a otros cuatro más, a usar el lenguaje de signos norteamericano para sordos como medio de comunicación. Washoe pudo adquirir un vocabulario de 132 signos con los cuales fue capaz de formar nuevas combinaciones.

Premack (1976) enseñó a Sara, un chimpancé, a seleccionar algunas fichas plásticas que representaban palabras inglesas. Sara fue capaz de seleccionar una ficha específica del conjunto y organizar una secuencia de cuatro fichas. Sara había aprendido a diferenciar una ficha plástica sin significación de una con significación que utilizaba como una "palabra". Los experimentos de Premack demostraban que Sara no diferenciaba las fichas por sus propiedades físicas sino más bien por sus propiedades semánticas y que el animalito tenía capacidades lógicas para desarrollar la comunicación lingüística.

Los trabajos de los Gardner y de Premack han sido criticados, porque en el sistema de comunicación la "sintáxis" está ausente.

Para superar estas críticas, Rumbauhg, Gill, Van Glaserfeld, Warner y Pisan (1975) crearon un lenguaje artificial: el Yerkis. Lana, una chimpancé podía obtener cuanto era necesario para vivir en buenas e interesantes condiciones. Las 24 horas del día pudo manipular el teclado del computador que corrientemente tenía 75 teclas pero podía aumentar el número a 125. En cada tecla había una configuración geométrica o lexicograma que representaba una palabra específica. Lana comunicaba mediante una selección apropiada a lo que quería "decir" 
y las organizaba en una sentencia aceptable de acuerdo en el lenguaje "Yerkis". Lana podía hablar y leer. Era capaz de expresar sus deseos, pedir cosas que nos estaban a la vista, preguntar por el nombre de los objetos nuevos. Aprendió a decir "sí" y "no".

Ella fue capaz de decir al experimentador que abandonara la pieza, porque a ella no le agrada su presencia $y$, varias otras cosas.

Aún más Sue Savage-Rumbaugh, Rumbaugh, Boysen (1978) demostraron que existe comunicación simbólica entre dos chimpancés y los mismos autores dan claras evidencias (1980) de la habilidad de los chimpancés para pensar, usar del lenguaje y evocar situaciones no presentes en el momento actual.

La actividad social de diversas especies de animales es conocida. Esta actividad es de gran desarrollo en los primates no humanos, de Waal (1990) (Project Aruhem) en el análisis de las relaciones en grupos de chimpancés al tratar el problema de los "Mecanismos Sociales" describe la dinámica de los "procesos políticos" que se desarrollan en un grupo de estos primates estudiados por él y sus colaboradores. La convivencia intragrupal estaría basada en una serie de fenómenos psicológicos. La "dependencia del rango" que permite a los individuos operar independientemente de sus capacidades individuales, respaldado por el estatus de sus familiares y amigos. Incluye el autor fenómenos como el significación de la "jerarquía de las hembras", el comportamiento social, que el denomina "inteligencia estratégica", las diferencias de estatus, "diferencias entre los sexos" y el "reparto". El "reparto" es una institución que tiene un fuerte significado en la dinámica social. Aún cuando pueda haber una disputa por alimentos. Hay siempre alguien que interviene, toma el alimento, generalmente hojas, lo reparte y el resto lo devuelve al disputante que había encontrado las hojas.
Otro aspecto de la vida social de los animales es la actividad lúdica, la cual probablemente es una actividad característica de todos los vertebrados $\mathrm{y}$, sin duda, de todos los mamíferos.

Hassenstein (1980) sostiene que el juego integra comportamientos aprendidos y genéticamente determinados, es más intenso en las épocas infanto-juveniles, una época de exploración, curiosidad e imitación de la vida adulta. El juego genera una experiencia que puede ser transferido a la vida adulta.

Los animales domésticos, perros, gatos entre otros, tienen grandes habilidades lúdicas.

Feddersen-Petersen (1987, pág.45-49) ha escrito un concienzudo análisis del juego en el perro, donde describe diversos tipos de juegos, entre otros el juego del "ladrido", el juego de "traer la pelota", el juego de "arrancarse", el juego de "morder en broma", etc. Menciona una postura lúdica en la cual el animal baja la mitad anterior del cuerpo, los codos están fuertemente flextados, las extremidades anteriores permanecen separadas, la cabeza eventualmente inclinada o moviéndose de atrás-adelante. En estas condiciones el perro puede ladrar. De esta postura el animal puede saltar y arrancarse, mirando hacia atrás, como un invitación a un juego de correteo. Bekoff (1995), llama a esta conducta, "conducta de reverencia".

El uso de herramientas y de otros artefactos ha sido descrito frecuentemente en primates, aves, cefalópodos y otros. Una observación que hemos hecho en las costas chilenas del Norte, en relación al comportamiento de las gaviotas, es la siguiente. Las aves revoloteaban a poca altura de la región de la costa donde el agua, después de reventar la ola, baña la superficie arenosa convirtiéndola en un espejo. De pronto se lanzaban con mucha velocidad hacia la arena húmeda y se elevaban de nuevo. Luego soltaban algo que se estrellaba contra la arena 
endurecida por el agua. Bajaban, tomaban lo que habían arrojado sobre la arena y volvían a repetir la operación hasta que el artefacto que habían dejado caer se rompía. Volvían a bajar, tomaban algo y no volvían. Observamos que el objeto que lanzaban repetidamente era un marisco bivaldo, el cual al romperse proveía de alimento a la diligente ave. Más aún observamos que muchas veces había otras gaviotas posadas cerca del lugar donde caía el marisco, que como nosotros, observaban atentamente. Estas observadoras no estaban interesadas en aprender una nueva tecnología alimenticia, sino en engullir el producto del trabajo de las trabajadoras. Se lanzaban rápidamente sobre el producto liberado por la destrucción de la concha, lo cogían antes de la llegada de la productora y se perdían de nuestra vista. Esta observación incide sobre dos aspectos del conocimiento adquiridos por las gaviotas: una tecnología productiva y una forma de explotación. Hunt (1996) ha descrito utilización de herramientas por parte de cuervos para recolectar su alimento, o el uso de piedras que realiza el buitre egipcio para romper huevos de avestruz.

Respecto al problema de la autoconciencia, Tattersall (2002, p.56-78) distingue dos aspectos del problema: el autoconocimiento del autoreconocimiento. Sin lugar a dudas el conocimiento de sí mismo es una función ampliamente difundida entre los animales. Con respecto al reconocimiento de su propia imagen, reflejada en un espejo, la situación es diferente, mientras los humanos aprenden rápidamente a usar el espejo, los chimpancés y los orangutanes también aprenden, aunque tal vez con mayor latencia que los humanos.

Los gorilas que han sido probados no reconocen su propia imagen, salvo excepciones. En cambio los monos no tienen esa habilidad. Pero sí pueden reconocer en la imagen del espejo rincones de su medio ambiente $y$ a otros colegas.
Un problema que tiene relación con el conocimiento de sí mismo ha sido traído a discusión por experimentos de Leonardo y Konishi (1999). Estos autores han mostrado que una ave, el mandarino diamante es capaz de corregir su propio canto si este mediante un truco tecnológico es alterado y se engaña al ave haciéndole escuchar una versión incorrecta y falsa (por ejemplo, cuando ciertas sílabas han sido introducidas mientras emite su canto). El mandarino modifica la ejecución tratando de corregir las anomalías percibidas, al escuchar la versión incorrecta. Esta acción implica que el animal compara la versión alterada con la versión de referencia, registrada en su memoria. El engaño auditivo desemboca finalmente en una deriva progresiva del canto producido. Si le permite escuchar de nuevo su canto natural, el ave recupera su versión inicial después de algunos meses.

Partiendo de la base que todo proceso psicológico es el resultado de la interacción de los fenómenos comportamentales y subjetivos que se integran en el cerebro, debemos entender que el conocimiento es un fenómeno psicológico. El conocimiento es un importante factor en la adaptación de los individuos de diversas especies. Por lo tanto este fenómeno psicológico juega un rol en la evolución de las especies y no es exclusivo del Homo sapiens.

Veamos el caso de los cánidos como ejemplo de fenómenos psicológicos.

Darwin (1872) es de la opinión, que los perros como muchos animales, configuran una actividad corpórea que es específica para cada emoción.

Los perros, como los lobos, son seres que juegan tanto con los individuos de la misma especie como con miembros de otras especies. Los perros incluyen en sus juegos al Homo sapiens. Hay diferentes juegos como "perseguir-ser perseguido", "hacer caras", "ladrar", "saltar", "morder en juego", "luchar", "acarrear", que son entre los perros y los hombres. 
Un aspecto muy interesante de la psicología canina es la facilidad para entenderse con el Homo sapiens. Los perros tienen una gran habilidad para captar gestos, expresiones faciales, inflexiones sonora de ladridos, por otra parte tienen grandes habilidades para adquirir aprendizajes asociativos sean estos excitatorios o inhibitorios, instrumentales o clásicos. Todo lo cual les confiere una gran elasticidad adaptiva. Tienen, sin lugar a dudas, la capacidad "misteriosa" de resolver problemas que no necesariamente están en su conjunto de conocimientos, como por ejemplo encontrar el camino de retorno a casa desde un sitio no conocido previamente. Esta propiedad psicológica facilita las interacciones individuales hombre-perro.

Frente a la cuestión de si el perro aprehende o empatiza con los estados de ánimo, o sentimientos de su dueño, la respuesta de todos los que normalmente viven con perros es afirmativa. "Los perros pueden diferenciar los estados de ánimo de los hombres y reaccionar "adecuadamente". Sin embargo los perros seguramente no tienen acceso a los contenidos informacionales de estos estados de ánimo, pero los estados de ánimo de sus amos actúan sobre los animales como una "transferencia contaminante". (FeddersenPetersen, 1987 p. 61). Esto obviamente implica el reconocimiento de la existencia de procesos subjetivos en el perro. Lorenz (1942) es del parecer que los perros tienen vivencias subjetivas similares a los humanos.

Es sabido que los animales son capaces de resolver problemas sin que ello implique una transferencia de conocimientos. Son clásicas las observaciones que hemos mencionado de Köhler (1925) en su chimpancé que resolvía problemas que obviamente demandaban una actividad subjetiva lógica.

Es conocido que diferentes especies aprenden por imitación. Hemos realizado observaciones en las cuales una monita (Macaca mulata) hacía esfuerzos por abrir la puerta de su jaula, mientras los vecinos de las otras jaulas observaban. La técnica de abrir esas jaulas de socializó una vez que ella aprendió como hacerlo.

Pero no solo los vertebrados aprenden por imitación. G. Fiorito y P.Scoto (1992) realizaron experimentos con Octopus vulgaris. En presencia de otros pulpos ("observadores"), un ejemplar ("demostrador") fue sometido a una prueba diferenciación condicionada en la que una bola roja al ser elegida se asociaba a una recompensa y, una bola blanca, a un castigo. El demostrador aprendió rápidamente a elegir la señal condicionada positiva. Los "observadores" al ser puesto en la situación de la prueba eligieron en el primer ensayo la bola roja.

Estos aprendizajes por imitación son una buena muestra de la participación de los procesos subjetivos en la conducta de los animales, donde el proceso percibido es transformado en un proceso virtual, almacenado en la memoria reciente y la evocación de la memoria usado como modelo efector.

El siglo XX ha sido muy rico en descubrimientos que dan cuenta del rol del sistema neuroendocrino en el control, tanto de las funciones comportamentales como de las subjetivas. Lo cual significa conferir al cerebro un rol fundamental en la génesis, consolidación y aplicación de los conocimientos como la ha hecho notar González Martín (1975).

La integración de las técnicas de investigación psicológicas con las técnicas en uso por la neurofisiología han permitido un desarrollo considerable en el conocimiento de los procesos gnósticos, los cuales, dicho de una manera sintética, se refieren al "saber que" y al "conocer como".

A las técnicas quirúrgicas, electrofisiológicas y farmacológicas que de alguna manera no abordaban completamente la dinámica de funciones estudiadas que ocurren en una dimensión temporal, se han agregado las técnicas de "emisión de positrones" y las del análisis de los "campos magnéticos" que 
se producen en zonas específicas del tejido nervioso comprometidas en la función considerada. Una buena descripción de éstas técnicas se encuentra en el trabajo de Posner y Raichle (1994) que han encontrado numerosas aplicaciones en la tecnología clínica.

Un aspecto nuevo ha surgido del estudio del significado funcional que tiene la adquisición de conocimientos o si se quiere el ejercicio del conocimiento. Es un hecho clásicamente aceptado que la constante práctica de una actividad mejora grandemente la eficiencia de los individuos en el ejercicio de esa actividad. La regla parecía ser válida para el Homo sapiens y para diversos animales. El entrenamiento favorece, por ejemplo la capacidad de discriminar frecuencias auditivas, en los límites extremos de las frecuencias audibles, perfecciona las respuestas motoras haciéndolas mas ajustadas a sus finalidades, facilita el reconocimiento de objetos, etc. Todo implica al menos cambios plásticos del Sistema Neuroendocrino (SNE).

\section{Discusión}

En términos generales el problema del conocimiento animal tiene que ser estudiado en el contexto adaptativo considerado tanto filo como ontogenéticamente. Las actividades gnósticas están básicamente orientadas por la interacción "individuo-medio"- Este es una función específica del SNE, la cual consiste básicamente en recoger información a través de las diversas modalidades sensoriales envueltos en la captación de aspectos discretos del medio, y en codificar esta información en señales bioeléctricas que se transmiten a través de las vías aferentes alcanzando grupos neuronales responsables de las funciones gnósticas, afectivas, mnémicas, etc. Esta distribución varía según sea la actividad concreta que el animal emprenda, los cuales en términos generales activan procesos subjetivos y comportamentales (Neisser (1976), Schettleworth (2001), Boysen e Hilmes (1999).)
Konorski (1967) hace un lúcido análisis de la existencia de las unidades gnósticas responsables de los fenómenos preceptúales ligados a las diferentes modalidades sensoriales, formando los así llamados campos gnósticos para las diferentes modalidades, como la intervención de las unidades efectoras responsables de los actos motores y también proposiciones hipotéticas de la génesis de las imágenes y alucinaciones. Es decir es un completo análisis tanto de los fenómenos virtuales como de la actividad efectora del cerebro que en el curso de los años ha sido confirmada experimentalmente.

La disputa sobre la mayor o menor importancia de los conocimientos que se refieren al procesamiento subjetivo de la información o de aquellos referidos la propiedad de esta información de desencadenar una reacción efectora (la cual incluye al comportamiento), es una cuestión más bien académica. En parte es la consecuencia de usar una terminología que dificulta el análisis de los problemas. Los procesos subjetivos, como los efectores son las piezas claves de la génesis de los conocimientos y constituyen una preocupación de la etología comparada, de la psicología experimental y el aprendizaje, en una perspectiva filogenético y ontogenética. (Neisser 1976, Boysen e Hilmes 1999, Schettleworth 2001).

Al hablar de conocimiento animal y de conocimiento humano, la primera pregunta que surge es que entendemos por "conocimiento". Se sostiene que las conclusiones están centradas en el proceso subjetivo (cognition) como tal antes que en los contenidos de las experiencias objetivas (Wasserman 1993) sin hacer ninguna alusión a la conciencia (Shettleworth, 2001).

El sistema neuroendocrino, específicamente el cerebro, es un órgano que ha evolucionado como consecuencia de la evolución de las especies y ha tenido como función regular los procesos adaptativos. 
Konorski (1967) al referirse a las actividades básicas de los organismos sostiene que el sistema nervioso de los animales más evolucionados tiene dos propiedades fundamentales, la reactividad y la plasticidad. Describe la reactividad como la capacidad de este sistema de ser activado por la estimulación de los receptores y la plasticidad como la capacidad del sistema de cambiar sus propiedades reactivas como resultado de sucesivas activaciones. Konorski considera también en el análisis de las funciones cerebrales, la generación de datos introspectivos o mentales, los cuales emergen de una tercera función integrativa del sistema nervioso que hemos designado como la "subjetividad", que es la capacidad del sistema nervioso de generar o convertir en representaciones virtuales los estímulos o las informaciones obtenidas del medio externo o del medio interno (Santibáñez- H, 1986,1991, 1999).

La idea de una secuencia de actividades integradas siguiendo la evolución de las especies aparece con mucha claridad cuando se mira con cuidado un cronograma de la evolución de las especies. Hagamos una incursión muy insuficiente de esta secuencia temporal.

- Sólo hace 2,5 billones de años que aparece una pequeña cantidad de oxigeno en la atmósfera terrestre. Entre 1 billón y 500 billones de años han aparecido los primeros metazoos, las lombrices segmentadas, arrecifes de corales y de peces, ancestros de arañas e insectos, trilobites, pequeños bivalvos, equinodermos, artrópodos, etc. Podemos inferir este grupo de animales tendría una actividad integrativa predominante, la reactividad.

- En cambio en el periodo que va entre los 500 y 100 millones de años con el desarrollo de estructuras nerviosas complejas hizo su aparición una función nueva: la plasticidad. Entre los nuevos integrantes de la naturaleza habríamos encontrado, vertebrados, insectos, calamares, anfibios, reptiles, aves, mamíferos, tortugas, dinosaurios, marsupiales, ancestros de las caballos y de las vacas. Todos estos animales aún en la actualidad tienen la habilidad de aprender.

- Entre 100 y 12 millones de años una hipótesis de Luis Álvarez y otros sostiene que la tierra fue alcanzada por un meteorito que causó una terrible catástrofe ecológica. Después del meteorito se encuentran fósiles de aves modernas, mientras que los restos de aves dentadas primitivas no habrían sobrevivido a la catástrofe ecológica.

En este periodo se inicia el desarrollo de mamíferos, de marsupiales y placentados, de peces con esqueletos óseos. Hacen su aparición las primeras evidencias de ancestros humanos en el Este Africano. Aparecen las líneas ancestrales de pongidos (simios) y de hominidos. Se encuentra en Uganda un antecesor común de hombres y simios Morotopithecus biskopi. Probablemente estamos frente a un periodo en el cual los procesos plásticos se encuentran bien desarrollados y aparecen los primeros esbozos de la actividad subjetiva.

- Entre 12 millones y 30 mil años tienen lugar cambios evolutivos que acercan el panorama ecológico a nuestros días y la estructura del sistema neuroendocrino a la nuestra. Este es el periodo en el cual aparece con mayor claridad la fase en la cual los procesos subjetivos adquieren primordial relevancia en la adaptación de primates especialmente del género Homo.

- A 2,5 millones de años comienza el paleolítico con las primeras herramientas hecha por el Homo abililis. En este mismo periodo se produce una glaciación que extiendo los hielos de la Antártica. África experimenta una sequía, que reduce las zonas boscosas y que convierte inmensas extensiones en pastizales. Las excavaciones de esta época muestran un pique de la extin- 
ción y la aparición de nuevas especies. En este periodo la línea de los hominidos se bifurca: una rama a los robustos y la otra al hombre moderno.

- Alrededor de 400-300 mil años atrás habría aparecido el lenguaje articulado. Se hizo posible como sugieren estudios realizados en cráneos del Homo erecto (Homo sapiens arcaico) que han permitido establecer la movilidad de su laringe.

- Hace 28 mil años hace su aparición el Homo sapiens - sapiens antes de lo cual se han encontrado 10 diferentes representantes del género Homo (Crubézy y Broga 2003).

Un análisis antropológico de la evolución de las especies permite llegar a algunas conclusiones, tal vez de interés si se consideran conjuntamente con la evolución de los fenómenos psicofisiológicos. Esto es comprensible si se tiene en cuenta que el cerebro, el sistema neuroendocrino en su conjunto, es una pieza fundamental en la evolución de los seres vivos. Más aún, si las habilidades adaptativas son la base de la sobrevivencia de los seres vivos. Desde la perspectiva filogenético la tendencia de los seres vivos a adaptarse es una actividad que conduce permanentemente a los seres vivos a mantenerse en vida. Metafóricamente podemos calificar a la adaptación como una "motivación vital" permanente, lo cual nunca alcanza un nivel de saturación o de "saciedad". Permanece activa a través de la historia vital de los seres vivientes.

En el curso de la filogenia han aparecido, en secuencia, los fenómenos reactivos, los plásticos y los subjetivos. Esta es una secuencia integrativa que ha estado en la base de la adaptación.

La reactividad ha sido la función que ha permitido la adaptación en los primeros escalones del desarrollo de las especies. La reactividad es una habilidad "responsiva". En concreto es una respuesta al estímulo, si- tuación o fenómeno que actúa por presencia. La reactividad es un proceso que confiere mayor eficiencia adaptativa, aunque dentro de ciertos límites.

La reactividad a través de la experiencia de las especies se ha transformado en plasticidad. La experiencia transforma la reactividad en un fenómeno de aprendizaje. El aprendizaje tiene una mayor eficiencia adaptativa que la reactividad. El aprendizaje tiene un carácter "preparatorio". Presenta no obstante un inconveniente limitante. La conexión, el nexo, la asociación o el enlace entre la situación que prepara y la situación relevante requiere tiempo. Queda entonces abierta la posibilidad de escapar, cosa que es mucho más difícil en la alternativa de la reactividad, cuando la respuesta adaptativa debe evitar una situación determinada.

Con el desarrollo que el sistema neuroendocrino ha alcanzado en la historia evolutiva de los seres vivos, se ha generado una tercera actividad integrativa, la "virtualización o subjetivación" de la realidad. Esta capacidad se ha integrado con las dos anteriores aumentando considerablemente las habilidades adaptativas de las especies que las poseen. La importancia de los procesos subjetivos en la adaptación y sobrevivencia de las especies que los poseen consiste en la particularidad de ser un fenómeno real - actividad de las neuronas - que tiene la propiedad de representar los objetos reales al margen del tiempo y del espacio. De este modo la realidad puede modelarse "a priori" en su versión "virtual"

Ahora bien la adaptación, es decir la propiedad de los seres vivos de subsistir en determinadas condiciones ecológicas, implica una interacción permanente entre el medio externo, el medio interno y el medio subjetivo. Todo medio es una instancia variable, la adaptación de un ser vivo es una función que compromete a los tres medios mencionados. Si las variaciones de una de los medios so- 
bre pasa los límites compatibles con la adaptación, esta se dificulta. Eventualmente puede crearse una condición que se hace incompatible con la sobrevivencia. C. Bernard describió la homoestásis del medio interno como una condición necesaria para la mantención de la adaptación. De la misma manera podemos hablar de una homeoestásis del medio subjetivo (Santibáñez - H, 2002), la cual puede alterarse por una virtualización de procesos alterados de los medios objetivos o por un desequilibrio funcional del sistema neuroendocrino. Desde esta perspectiva la adaptación puede operar en varios niveles funcionales, en los cuales se pueden integrar las contribuciones de Waddington (1952, 1954,1961), con las de Marais (1973), Pavlov (1954) y Konorski (1968) permiten distinguir varios tipos de adaptación.

Waddington distinguió tres tipos de adaptaciones:

1. Adaptación exógena incluye fenómenos que modifican el comportamiento para obtener una adaptación que responsa a los requerimientos específicos de determinadas situaciones ambientales. Esto puede incluso conducir a que si una rata al nacer es amputada de las extremidades anteriores, el animal aprende a usar sus miembros posteriores, desplazándose en una posición bípeda. Esta situación corresponde a una modificación de la reactividad, (Konorski), al desarrollo de un reflejo condicionado (Pavlov) y al uso de una actividad controlada por la memoria causal (Marais).

2. Adaptación endógena accionada por procesos funcionales genéticamente determinados, que el animal trae consigo desde el nacimiento, los cuales le permiten ajustarse instintiva y automáticamente al medio. Estos fenómenos implican la memoria filética (Marais) basada en la reactividad (Konorski) que constituyen los reflejos incondicionados (Pavlov).
3. Adaptación pseudoexógena en la cual el animal exhibe rasgos similares a los exógenos, pero su origen es hereditario, independiente de la influencia ambiental.

Estas observaciones de Waddington permiten establecer una relación directa y natural de los fenómenos de adaptación y la actividad integrativa del sistema neuroendocrino.

Los conceptos de "asimilación" y "canalización" propuestos por Waddington consecuencia de la plasticidad del genotipo en respuesta a la variabilidad ambiental, tienen probablemente lugar gracias a las funciones del sistema nervioso. En consecuencia no se puede excluir el influjo de los fenómenos subjetivo en la adaptación, específicamente en el ajuste individuos al medio.

Con respecto al conocimiento hay que dejar en claro que esta función psicológica es un proceso "comportamental-subjetivo", tanto filo como ontogenético si se considera la evolución o el desarrollo. En las etapas primarias de ambos procesos aparecen primero la componente efectora y luego la subjetiva.

El conocimiento se va generando en la medida que los individuos de una especie determinada van enfrentando una situación nueva, la cual tiene necesariamente que ser confrontada.

Todo conocimiento resulta de una función del Sistema Neuroendocrino llevada a cabo para adquirir el conocimiento. Esta actividad ha sido calificado por nosotros como "gnoseogénesis" (Santibáñez-H, 2001).

La interacción gnóstica implica directamente la asimilación de diversas propiedades del objeto que quien conoce o intenta conocer pueda captar sea por acción directa o indirecta del objeto sobre la superficie receptora del sujeto o por la observación de los efectos que el objeto produce sobre otros objetos o por el análisis de las huellas que deja el objeto. 
El conocimiento se genera en diversas etapas por acumulación de unidades gnósticas o "cgnits" (Fuster 2002).

La primera etapa del conocimiento es la habilidad de reconocer el objeto, es decir la localización del objeto en el espacio y el tiempo. Esta función se genera con la percepción.

Anojin (1962) sostiene que el conocimiento confiere, a quien conoce, la propiedad de anticipar el efecto de una acción suya que opere sobre el objeto. Esto demuestra que los procesos gnósticos son una reflexión o representación de la realidad conocida. Los procesos de aprendizaje que operan sobre la realidad a través de sus representaciones subjetivas son una prueba de la acción anticipatoria del conocimiento y de la equivalencia funcional entre el objeto de conocimiento y su reflexión subjetiva.

Anticipación, predicción y previsión son propiedades que los conocimientos confieren a quien o quienes conocen. Una hipótesis es una pregunta que un científico se hace en la búsqueda de condiciones anticipatorias que le permitan modelar un tipo de conocimientos. A medida que se va enriqueciendo el acervo de anticipaciones a las manipulaciones anticipatorias, se va ampliando el campo gnóstico de este objeto en el medio subjetivo del ser vivo que conoce y en la praxis que desarrolla frente a este objeto.

La anticipación, produce un incremento del campo gnóstico que se manifiesta en una nueva etapa del "control" del objeto de conocimiento. En esta etapa, se está en condiciones de ejercer influencias sobre el objeto conocido lo cual permiten orientar sus procesos dinámicos en una dirección determinada por el sujeto.

La última etapa del conocimiento posibilita a las entidades cognoscentes crear, reproducir, modificar al objeto conocido.

El proceso gnoseogénico empieza por la habilidad de reconocer al objeto y termina con la capacidad transformarlo, reproducirlo, recrearlo.

El conocimiento no es un fenómeno abstracto. Es una actividad concreta que se desarrolla en marcos espacio-temporales de duración variable pero limitada. Es además concreto, porque los objetos que activan los procesos gnósticos son de la más variada naturaleza y en consecuencia las formas operatorias gnósticas son también muy variables. Como todo fenómeno psicológico es una actividad sostenida por fuertes estados motivacionales de diversas índoles que modulan tanto el comportamiento de los individuos como de los grupos de individuos que lo generan.

El conocimiento es acumulativo, claramente en el transcurso de la evolución, a través de la herencia cultural y tal vez, de la herencia genética. El conocimiento se genera de una manera episódica, cada ciclo empieza en la percepción del objeto y termina recién en la última etapa en la reproducción del fenómeno procesado gnósticamente.

La duración de un ciclo gnóstico es variable. Depende de una serie de factores que van desde la accesibilidad del objeto al procesamiento gnóstico, a los instrumentos necesarios para llevar a cabo el análisis gnóstico sin dejar de lado las condiciones psicológicas de quien o quienes realizan esta tarea.

Vistas así las cosas, puede aceptarse que el proceso gnoseogenético está formado por una gradiente gnóstica que se va integrando con el progreso de los sucesivos logros gnósticos. Esta dinámica vale tanto para el conocimiento empírico como el epistémico, para el género Homo como para otros géneros.

El sistema Neuroendocrino, especialmente el Encéfalo, es el órgano del conocimiento. Sin cerebro no hay conocimiento ni procesos psicológicos. La producción de conocimientos puede asimilarse a procesos de aprendizaje, los cuales tienen diferentes grados dificultad: algunos son fáciles de apren- 
der, otros requieren un largo proceso de entrenamiento.

Sin embargo sería ingenuo pensar que el SNE adquiere la habilidad de conocer independientemente del medio con el cual interactua. Fuera de la interacción individuomedio, fuera del contexto psicológico, las funciones neurales subyacentes al conocimiento decaen y el tejido nervioso afectado no se desarrolla o degenera. Este punto esta probado por todos los experimentos de deprivación sensorial o de sistemática inhibición funcional, o por el efecto de la experiencia temprana. Se conoce, desde hace tiempo, que la deprivación forzada de la actividad receptiva de los organismos lleva a la perdida de la eficiencia senso-perceptiva de la modalidad afectada. En condiciones normales todos los felinos dominan los movimientos necesarios para caminar con muy poco esfuerzo, pero cazar es un conocimiento que se adquiere con un aprendizaje más empeñativo, especialmente para aquellos felinos que como los leones organizan socialmente sus cacerías.

La interdependencia entre el funcionamiento del sistema neuroendocrino y los procesos psicológicos, gatillados por el medio ambiente presentan algunos fenómenos interesantes como la neurogénesis secundaria que tiene lugar después de superada la etapa embrionaria que es responsable de la neurogénesis primaria.

Es conocido que los canarios machos al llegar la primavera cantan más intensamente que en otras estaciones, para atraer las hembras. Estos cantos cambian anualmente debido a que los animalitos imitan los cantos de otros machos de la vecindad. El mero canto una vez asimilado es retenido y ejecutado sin ninguna alteración audible.

Notebohm $(1989,2000,2001)$ identificó los grupos neuronales responsables del aprendizaje y ejecución de estas interpretaciones que forman una estructura que llamó "centro vo- cal superior". Observó que en el macho de esta especie hay tipos de neuronas: auditivas que escuchan las canciones, y motoras que operan la musculatura de la laringe (órgano emisor de sonidos) previa sinapsis con dos estructuras diferentes: con un núcleo premotor (RA) y de ahí al núcleo motor XII par que inerva los músculos de la syringe.

El canto de estos animalitos sufre un periodo de alteración antes de alcanzar el equilibrio que presentará en primavera. El autor mencionado y sus colaboradores mostraron que en ese periodo de transición hay una degeneración de neuronas pertenecientes centro vocal superior, pero al mismo tiempo hay un incremento de neuronas nuevas. Al núcleo RA llega una nueva serie de neuronas que se han formado en la zona subventricular del ventrículo lateral y de ahí han emigrado al centro vocal superior, lugar en el cual no todas estas neuronas nuevas sobreviven. (Lois y Álvarez-Buylla, 1993 y Álvarez-Buylla y García Verdugo, 2002). Del centro vocal superior parten dos grupos neuronales, los recién formados que sobreviven y otros que son permanentes, ambos grupos se integran en RA. La sobreviva periódica de las células conformadas esta condicionada por un factor que los autores han llamado "Factor neurotrófico derivado del cerebro" (FNDC). La testosterona favorece la producción de este factor y a su vez ella es facilitada por la presencia de luz, luminosidad diaria, que aumenta en primavera cuando se produce la renovación del repertorio. En otoño la concentración de testoterona disminuye lo cual hace bajar la producción del factor neurotrófico y la degeneración neuronal aumenta. El cambio anual de repertorio responde al cambio estacional que afecta a los canarios.

Fenómenos similares han sido descríto por Goddard (1991). El autor sostiene que las capacidades de crear información y mantenerla por largo tiempo en la memoria se ha desarrollado primariamente en animales no pertenecientes al género Homo, específi- 
camente el aprendizaje de las vocalizaciones y las formas de adquirir alimentos. Estas funciones psicofisiológicas definen los padrones sociales, la habilidad de reconocer tanto las sociedades animales como las humanas.

En este último tiempo han aumentado los estudios que apuntan a la neurogenesis en seres vivos adultos que posibilita el recambio neuronal aún con el objetivo de reparar o reemplazar las neuronas disfuncionales. El hipocampo adulto produce las células madres que luego una vez emigradas a las estructuras pertinentes se convierten en neuronas normalmente funcionantes.

Otros investigadores han hecho descubrimientos similares en mamíferos. Se ha puesto en evidencia la existencia de una neurogénesis secundaria en el sistema olfatorio de roedores adultos, en particular en el bulbo olfatorio, el hipocampo y la corteza (Lois y Álvarez-Buylla, 1993). Gheusi et al. (2000) mostraron claramente que el aumento de neuronas modifica el comportamiento olfatorio de las ratas.

Álvarez-Buylla y García-Verdugo (2002), estudiando la neurogénesis adulta constataron que en la zona subventricular tienen origen las células precursoras de las nuevas neuronas. De allí emigran a las estructuras olfatorias correspondientes donde al ubicarse entre las neuronas de estas estructuras se transforman en neuronas.

Erikson et. al (1991) estudiando cerebros humanos ha buscado en los sitios que en roedores y monos tiene lugar la formación de células precursoras de las neuronas después de emigrar. Han encontrado evidencias claras que sugieren que en el hombre tiene lugar el proceso descrito, es decir neurogenesis.

Kemperman (2002) sostiene que la neurogénesis es afectada por el comportamiento, especialmente en lo que respecta a funciones hipocampicas relacionadas con el comportamiento. Además que las funciones en las cuales interviene la neurogénesis se ven favorecidas por esta.

En síntesis. El conocimiento es una función que resulta de un proceso de interacción entre animales y sus respectivos medios externos. Esta interración esta mediada por el Sistema Neuroendocrino que es el órgano de procesamiento informacional y al mismo tiempo el órgano que permite al individuo reaccionar frente al objeto que provee la información.

Esta interacción permite al animal comportarse en función de las exigencias del ambiente. Pero al mismo tiempo modificar sus propias condiciones de reacción. Esta acción del medio sobre el SNE no sólo hace más eficientes las reacciones operantes sobre el medio, sino que llega en determinadas circunstancias a producir un recambio neuronal, que se conoce como neurogénesis post-embrionario.

\section{Referencias Bibliograficas}

Alvarez-Buylla, A., Kirn, J. R. \& F. Notтевонм, (1990). Birh of projection neurons in adult avian brain may be related to perceptual or motor learning. Science. 249: 1444-1446.

Alvarez-Buylla, A. \& M. García Verdugo, (2002). Neurogenesis in adult subventricular zones. J. Neurosci. 22(3): 629-634.

AnojIN, P. K. (1962). Reflexión anticipatoria de la realidad. (En Ruso) Vóprosy Filosofii. 7: 97-111.

BEKOFF, M. (1995). Play signal as punctuation: the structure of the social play in canids. Behaviour. 132: 419-422.

BREHM, A. E., Brehms Tierleben. 1863 (1926). Nach der zweiten Originalausgabe bearbeitet von Adolf Meiner. (17 Bände) Gutenberg-Verlag Christensen u. Co. Hamburg. 
Boysen, S. T. \& G. T. Hilmes (1999). Current issues and emerging theories in animal cognition. Anual Rev. Psychol. 50: 683-705.

Buytendij, F. (1928). Psychologie des Animaux. Payot. Paris. Pp. 315.

Crubézy, E. \& J. Braga (2003). Homo sapiens prend de l'age. La Recherche 368: 30-15.

DARWIN, CH. (1872). The expression of emotion in man and animals. John Murray. Albertmark Street, London.

Eriksson, P.; S. E. PerfileVA, BJÖRK-ERIKSON \& F. H. GAGE (1998). Neurogenesis in the adult human hippocampus. Nat. Med. 4(11): 1313-1317.

FABRE, J. H. (1946). Recuerdos entomológicos. 7 tomos. Emece Editores S.A. Bs. Aires.

Feddersen-Petersen, D. (1987). Hundepsychologie. Wesen und Sozialverhalten. Kosmos. Gesellschaft der Naturfreunde. Franckh'sche Verlagshandlung. Stuttgart, Pp. 60.

Fiorito, G. \& P. Sсото (1992). Observational learning in Octopus vulgaris. Science, 256: 545-547.

Fischel, W. (1961).Die Seele des Hundes. Parey. Berlin, Hamburg.

Fuster, J. (2002). Cortex and Mined Cognition. Oxford University Press. Pp 314.

Gardner, B. T. \& Gardner, R. A. (1969). Teaching sign language to a chimpanzee. Science 165: 664-672.

Gazzaniga, M. S. (1959) The Cognitive Neurosciences. A Bradford Book. The MIT Press. Cambridge, Massachusetts.

Gheusi, G., H. Cremer, H. Mclean, G. Chazal, J. D. Vincent, \& Lledo, P. (2000). Importance of new generated neurons in adult olfatory bulb for odor discrimination. Proc. Nat. Acad. Sci. 97: 1823-1824.
GODARD, R. (1991). Long term memory of individual neighbors in migratory songbirds. Nature. 350: 228-229.

Gonzalez Martin, D. (1975). El cerebro cognoscente: un modelo para su estudio. Academia de Ciencias de Cuba. La Habana. Pp. 324.

GrIFFIN, D. R. (1976). A possible window on the mind of animals. Amer. Sci. 64: 530- 535.

Guillaume, P. (1947). La psychologie animale. Librairie Armand Colin. Paris. Pp 211.

Hassenstein, B. (1980). Instinkt,Lernen, Spielen, Einscht. Piper-München.

Hayes, K. \& C. Hayes (1971). Higher mental function of a home raised chimpanze, In: A.M. Schreier and F. Stollnitz (eds), Behavior of a non human primate. Academic Press, New York and London. Pp. 312 - 356.

Healy, S. D. \& J.R. KREBS (1993). Development of hippocampal specialization of a foodstoring bird. Behav. Brain Res. 53: 127-131.

Hunt, G. R. (1996). Manufacture and use of hook-tools by New Caledonian crows. Nature, 379:, 249-251.

KempermanN, G. (2002). Why new neurons? Possible functions for adult hippocampal neurogenesis. J. Neurosci. 22 (3): 635-638.

KöHler, W. (1925). The mentality of apes. Harcourt, Brace. New York. Pp. 108.

KONORSKI, J. (1948). Conditioned reflexes and neuron organization. Cambridge: Cambridge University Press Pp. 270.

KONORSKI, J. (1967). The integrative activity of the brain. An interdisciplinary approach. Chicago, London. The University of Chicago Press. Pp. 350

KRALL, K. (1912) Denkende Tiere Leipzig.

KrechevsKI, I (D. KRECH)(1932). Hypothesis in rats. Psychol. Rev. 38: 506-532. 
KRECH, D. \& R. S. CRUTChFIELd (1948). Theory and problems of social psychology. McGrawHill. New York. Pp. 320.

Leonardo, A. \& M. Konishi (1999). Decrystalization of adult birdsong by perturbation of auditory feedback. Nature 399: 466-470.

Lois, C. \& A. Alvarez-Buylla (1993). Proliferating subventricular zone cells in the adult mamalion forebrain can differentiate into neurons and glia. Proc. Nat. Acad. Sci. 90 : 2074-2077.

LoRENZ, K. (1942). Die angeborenen Formen möglicher Erfahrung. Zeitschrift f. Tierpshychologie 5 : 235-409.

Marais, E. 1903 (1973). The soul of the apes. The soul of the white ant. Penguin Book. Pp. 314.

NeIsser, U. (1976). Psicología cognoscitiva. Editorial Trillas, México. Pp.16.

Notтевонм, F. (1989). From bird song to neurogenesis. Sci. Am. 260 (2): 74-79.

Notтевонм, F. (2000). Why are some neurons replaced in adult brain? J. Neurosci. 22: 624-628.

Notтевонм, F. (2000). Neuronal replacement in adult brain. Brain Res. Bull. 57 (6): 737-749.

Pawlow, I, P. (1953). Über die Psychische Sekretion der Speicheldrüsen. Sämtliche Werke. Band III, 1. Akademie-Verlag. Berlin. Pp 23-35.

Pawlow, I. P. (1953). Die Naturwissenschaftliche. Erforschung der sogenannten Seelentätigkeít der höheren Tiere. Sämtliche Werke. Band I. Akademie-Verlag. Berlin, Pp. 41-54.

Pawlow, I. P. (1954). Physiologie der Grosshirnhemisphären. Sämtliche Werke. Band V. Pp. 329-369.
Pieron, H. (1941). Psychologie zoologique. Presses Universitaires de France. Paris. Pp 255.

Posner, M. J. \& M. E. Raichle (1994). Images of mind. Scientific American Library. Pp. 257.

PREMACK, D. (1976). Intelligence in Ape and Man. In N.J. Hillsdale, Lawrence Erlbaum Assoc. Pp. 239.

Rumbaugh, D. M., T.v. Gill, E. Von Glaserfeld, H. Warner \& P. Pisani (1975). Converstion with a chimpanzee in a computer-controlled enviroment. Biol. Psychol., 10: 627-640.

Savage-Rumbaugh, E. S., Rumbaugh, D.M. \& BOYsEn, S. (1978). Simbolic communication between two chimpanzees. Science 201: 641-644.

Savage-Rumbaugh, E. S., Rumbaugh, D.M., \& Boysen, S. (1980). Do apes use language? Amer. Sci. 68: 49-61.

SANTIBÁÑEZ- H., G. (1986). Principal functions of the neuroendocrine system: the functional basis of the integrative activity of the brain., In Introduction to the physiopathology of neurotic states, ed. by G. Santibañez-H and M. Lindemann. Leipzig. Pp.357.

SANTIBÁÑEZ - H., G. (1991). Subjetivität als die dritte Umwelt. Deut. Zeitschrt. für Philosophie 39: 464-476.

SAntibáñez - H., G. (2001). Bases psicofisiológicas de la gnoseogénesis. Rev. Psicol. U. Chile. 10: 151-176.

SANTiBÁÑEZ - H., G. (2002). Dialéctica de los procesos subjetivos. Rev. Psicol. U. Chile. 11 (1) : 65-90.

SECHENOV, I. M. 1863. (1965) Los reflejos condicionados. Academia de Ciencias. Museo "Carlos J. Finlay". Cuba. Pp.231 
Settleworth, S. J. (2001). Animal cognition and animal behaviour. Animal Behaviour, 61: 277-286.

TATTERSAL, L. (2002). The monkey in the mirror. Oxford University Press. Pp. 203.

ThorndiKe, E. L. (1911). Animal intelligence. Experimental studies. New York McMillan.

WaAL, F. De. (1990) Chimpanzee politics. The John Hopkins University Press. Baltimore and London. Pp. 227.

Waddington, C. H. (1953). The evolution of adaptation. Endeavour. 12: 47.

WadDington, C. H. (1959). In Evolution after Darwin. University of Chicago Centennial. University of Chicago Press. Pp. 381-402.
Waddington, C. H. (1961). Genetic assimilation. Advances in Genet. 10 : 257 290.

WARden, C. J., Jenkins, T. N. \& WARner L. H. (1940). Comparative Psychology. Le Ronald Press Company. New York. Vol. I y II.

Wasserman, E. A. (1993). Comparative cognition: beginning the second century of the study of animal intelligence. Psychological Bulletin 113: 211-228.

Wundt, W. (1874). Grundzüge der Physiologischen Psychologie. 3 Bände. Verlag von Wilhelm Engelmann, Leipzig. 